\title{
BENEFICIOS E IMPACTOS DEL TELETRABAJO EN EL TALENTO HUMANO. RESULTADOS DESDE UNA REVISIÓN DE LITERATURA
}

\author{
Benefits and impacts of telework in human talent results from a literature review \\ Martha Luz Benjumea-Arias \\ Magíster en Ingeniería Administrativa. Instituto Tecnológico Metropolitano. \\ Medellín-Colombia.marthabenjumea@itm.edu.co \\ Eliana María Villa-Enciso \\ Magíster en Ingeniería Administrativa. Instituto Tecnológico Metropolitano, \\ Medellín-Colombia.elianavilla@itm.edu.co \\ Jackeline Valencia-Arias \\ Estudiante de Artes Visuales. Instituto Tecnológico Metropolitano, \\ Medellín-Colombia. javalenca.a@gmail.com
}

\section{Cómo citar / How to cite}

Benjumea-Arias, M.L., Villa-Enciso, E.M. y Valencia-Arias, J. (2016). Beneficios e impactos del teletrabajo en el talento humano. Resultados desde una revisión de literatura. Revista CEA, 2(4), 59-73.

Recibido: 27 de febrero de 2016

Aceptado: 30 de mayo de 2016

\section{Resumen}

El teletrabajo es una modalidad laboral que está emergiendo por las bondades que representa para las organizaciones y sus colaboradores, asociado a otros beneficios como la disminución de la contaminación ambiental, los tiempos de desplazamiento y la movilidad en las grandes metrópolis. Es por esto que el objetivo del artículo es presentar los beneficios para la calidad de vida del trabajador de esta forma de organización laboral innovadora, a partir de una revisión de literatura. Algunos resultados indican que, con el logro de un aumento en la calidad de vida del talento humano, se redundará en una mayor productividad, competitividad e innovación en las organizaciones. Con respecto a la gestión estratégica del talento humano en las organizaciones, en lo que se relaciona con las nuevas tendencias de trabajo, como el trabajo en red o teletrabajo, no se logró identificar la existencia de marcos conceptuales establecidos que permitan estimular esta modalidad laboral.
Palabras claves: teletrabajo, gestión del talento humano, calidad de vida, impactos.

\begin{abstract}
Teleworking is a work modality that is increasing mainly because of the benefits it brings to the life of employees. It is also important to mention the benefits this type of work has in reducing pollution, time taken to travel to workplace and mobility problems in large cities. From a literature review, the objective of this paper is to present the benefits of this innovative form of work for the quality of life of workers. Some results indicate that increased productivity, creativity and innovation are achieved in organizations with increased quality of life of human talent. With regard to the strategic management of human talent in organizations as it relates to new trends of work, such as networking or telecommuting, it failed to identify the existence of established conceptual frameworks to encourage this type of work.
\end{abstract}


Keywords: telecommuting, management of human talent, quality of life, impacts.

\section{INTRODUCCIÓN}

En el actual mercado competitivo, donde la innovación se ha convertido en un factor de vital importancia, es necesario que las empresas entiendan cómo estimular y promover el potencial innovador y creativo de sus colaboradores. Al respecto, la contribución y el comportamiento individual son características importantes para asegurar el éxito de una organización, la cual debe estar alineada con las políticas y programas que promueve la gestión del talento humano a partir de la estructuración de su función, la definición de su objetivo y la asignación de recompensas por su contribución (MuñozDoyague y Nieto, 2012).

Durante las últimas dos últimas décadas se ha observado un gran interés por el desarrollo de la gestión del talento humano en las organizaciones, con el objetivo de estimular la innovación y creatividad, así como la eficiencia de los colaboradores. Dado que los objetivos de muchas de las políticas que integran la gestión de talento humano son la motivación y el fomento de actitudes y comportamientos alineados al éxito organizacional, su efectividad estará condicionada por las percepciones que de ellas tengan los individuos a los que se dirigen (Gibb, 2001).

En este sentido, si los empleados perciben que la organización invierte en ellos y los considera factores estratégicos, estos responderán con comportamientos positivos orientados a la consecución de los objetivos organizativos (Kuvaas y Dysvik, 2010). A pesar de los diferentes enfoques desde los que se ha abordado la gestión humana, existe consenso en la necesidad de establecer un sistema integrado y coherente de políticas de talento humano en los que se profundice en el análisis de configuraciones, a través del estudio de las características de sistemas integrados (Stavrou y Brewster, 2005; Verburg, Den Hartog y Koopman, 2007).

En este sentido, el propósito fundamental de este artículo es realizar un acercamiento conceptual sobre el teletrabajo, los beneficios e impacto de esta modalidad de trabajo en la calidad de vida del colaborador, así como una revisión del fenómeno en Colombia y la importancia de alinearlo a la gestión estratégica del talento humano. El artículo presenta, en primer lugar, un marco teórico que contextualiza el teletrabajo y sus antecedentes, así como los beneficios y limitaciones; en segunda instancia, se presenta la metodología utilizada para realizar la revisión; posteriormente se presentan los resultados y la discusión de los mismos; por último, las conclusiones.

\section{MARCO TEÓRICO}

\section{Antecedentes del teletrabajo}

El concepto de teletrabajo hace referencia al trabajo a distancia, en el cual los empleados o trabajadores independientes laboran desde un lugar separado físicamente de las oficinas; esto se lleva a cabo a través de la telemática, que es la combinación del uso de las Tecnologías de Información y Comunicación (TIC), para desempeñar labores desde cualquier lugar y en cualquier momento (Mitter, 2000; Cárcamo y Villegas, 2009).

Al respecto, Haddon y Lewis (1994) plantean que el teletrabajo empezó a ganar mayor acogida en la década de 1970, a causa de la crisis energética. Esta modalidad laboral se llegó a considerar como una alternativa sustituta a los desplazamientos físicos, interfiriendo en el diseño urbano y las formas de vida de la población trabajadora. Estas razones llevaron a que en 1998 se diera un fuerte impulso al escenario casa-oficina, permitiendo a los empleados trasladar sus 
labores y funciones de lo industrial a lo local (Brosveet, 1997).

El uso de las TIC en el modelo de teletrabajo, se ha empleado como un indicador que muestra la formación entre la población respecto a la capacidad que tienen los empleados de adaptarse a nuevos cambios (Tregaskis, 2000; Meroño-Cerdán, 2016); así como aspectos y comportamientos de la comunicación familiar y organizacional (Lévy-Leboyer, 2003). Es así como esta modalidad laboral se convierte en una oportunidad para las empresas, ya que reduce costos de infraestructura y los empleados disminuyen sus gastos diarios y sus tiempos de desplazamiento (Julsrud, 2010).

El modelo de teletrabajo es visto como estrategia de generación de empleo y autoempleo, aspecto que motiva a los países a promoverlo y a regularlo. Sin embargo, no se aplica para todos los cargos en los que se hace necesaria la presencia física del trabajador en los procesos productivos. No obstante, se evidencia mayor aplicación del teletrabajo en profesionales como arquitectos, abogados, ejecutivos y el personal de ventas (Daniels, Lamond y Standen, 2001; Rodríguez, 2015) o empleados a los que sus funciones les permitan asistir por días al lugar físico de trabajo o laborar desde una oficina virtual que incluye computadores, módem y celulares (Mitter, 2000).

En América Latina, desde finales del siglo pasado, se ha experimentado una importante aceptación para la implementación del teletrabajo, debido a los avances en la tecnología de las comunicaciones y la información, el incremento de la productividad en las empresas, a la satisfacción de los empleados y a la disminución de los gastos relacionados con el transporte, vestuario, seguros del automóvil, combustibles, mantenimiento, estacionamiento, limpieza y alimentación, entre otros.

\section{El teletrabajo en Colombia}

Sobre la base de las consideraciones anteriores, el Ministerio del Trabajo, a partir de la Ley 1221 de 2008, mediante el Decreto 884 de 2012, sentó las bases para que las entidades públicas y privadas implementaran el teletrabajo. Dicha Ley tiene por objeto promover y regular el teletrabajo como un instrumento de generación de empleo y autoempleo, mediante la utilización de tecnologías de la información y las telecomunicaciones, con la seguridad jurídica que se resume en cinco aspectos que las empresas deben abordar: 1 . Voluntariedad del teletrabajo, 2. Acuerdo de teletrabajo, 3. Modificación del reglamento interno de trabajo, 4. Reporte ante la administradora de riesgos laborales, y 5 . Reversibilidad del teletrabajo.

Al respecto, el Artículo 2 de la Ley 1221/2008 define el teletrabajo como una forma de organización laboral, que consiste en el desempeño de actividades remuneradas o prestación de servicios a terceros utilizando como soporte las tecnologías de la información y las TIC para el contacto entre el trabajador y la empresa, sin requerir la presencia física del trabajador en un sitio específico de trabajo. Define también al teletrabajador como una persona que desempeña actividades laborales a través de tecnologías de la información y la comunicación por fuera de la empresa a la que presta sus servicios (Ministerio del Trabajo, 2008).

En Colombia, la Ley 1221 de 2008, considera tres formas de teletrabajadores: autónomos, móviles y suplementarios.

- Autónomos: labora en el domicilio o en un lugar escogido para desarrollar su actividad profesional (oficina, local comercial). Trabajan siempre fuera de la empresa y acuden en algunas ocasiones a la oficina.

- Móviles: no tienen un lugar de trabajo establecido. Las TIC y los dispositivos 
móviles son las herramientas primordiales para desarrollar sus actividades

- Suplementarios: laboran dos o tres días a la semana en la casa y el resto de tiempo en una oficina.

Según la encuesta realizada por el Centro Nacional de Consultoría de la Corporación Colombia Digital del Ministerio de las TIC, sobre una muestra efectiva de 1.740 empresas colombianas, al mes de agosto de 2016 el número de teletrabajadores se triplicó durante los últimos cuatro años, al pasar de 31.553 a 95.439. Bogotá cuenta con un número aproximado de 55.000 teletrabajadores, seguido de Medellín con 25.000 y Cali con 5.700. Al respecto, el teletrabajo se constituye en una oportunidad para que la población rural pueda beneficiarse en el marco del posconflicto (MinTIC, 2016).

Sobre el impacto, revela la encuesta que el $47 \%$ de las empresas consideran que el teletrabajo disminuye los costos operacionales representados en costos fijos, recursos, planta física y puestos de trabajo; el $47 \%$ aumento de la productividad; el $37 \%$ aumento en la eficiencia de los procesos; y el $41 \%$ que aporta a la sostenibilidad ambiental. En el caso de los empleados, el $49 \%$ perciben los beneficios del teletrabajo en razón del ahorro de tiempo en los desplazamientos y la movilidad; el $40 \%$ destaca su autonomía e independencia para desempeñar su labor; y el 33\% considera una mejora en calidad de vida (MinTIC, 2016).

\section{MÉTOdOLOGÍA}

Este artículo parte de un proceso de búsqueda y revisión de fuentes secundarias de información como camino para identificar tendencias y posibles vacíos en la literatura académica alrededor del teletrabajo. Esta revisión se estructura a partir de las siguientes etapas:
- La base de datos Scopus reporta 177 trabajos investigativos alrededor de la temática entre 1984 y 2016, siendo la revista New Technology, Work and Employment (Reino Unido) quien reporta un mayor número de investigaciones. Además, la última revisión de esta literatura se publicó en 2013, titulada: The survey of viewpoint of teleworkers in department of information processing about advantages and disadvantages of teleworking (Tahavori, 2014) y reportándose solo una publicación en español referente a una propuesta de un telecentro para ofrecer servicios profesionales vía teletrabajo (Blanco y Gil, 2014). Lo anterior es una muestra de la necesidad de generar mayor conocimiento sobre el teletrabajo, en artículos publicados en habla hispana y la necesidad de generar una revisión de literatura que permita entender los enfoques investigativos que se están dando a esta área de conocimiento.

- Definición de estrategias de búsqueda bibliográfica: con el fin de incluir literatura académica diversa en la temática durante el periodo comprendido entre 1995 y 2016, se definieron bases de datos académicas a nivel mundial (Scopus) y en Latinoamérica (Redalyc y Scielo), ya que era necesario contrastar los hallazgos en cada contexto. Se incluyeron en la búsqueda los artículos publicados en revistas indexadas, artículos de reflexión y actas de congresos y eventos entre que respondieran a la combinación siguientes palabras claves: teleworking, occupational mobility, residential mobility, teleworkers, teleworking policy, home-based telework, new forms of work, secure teleworking, home based business, flexible working.

- Evaluación y análisis de los estudios seleccionados: la estrategia de búsqueda realizada durante el último año dio como insumo una serie de publicaciones 
relacionadas con la temática, pero para la presente revisión se priorizaron los artículos que indagaran explícitamente los beneficios e impactos del teletrabajo en el talento humano. Como resultado, este artículo integra elementos de orden conceptual sobre sus antecedentes, el marco regulatorio en Colombia, los beneficios e impacto en la calidad de vida y su relación con la gestión estratégica del talento humano. Además, se incluye el resultado de la encuesta realizada por el Ministerio de las TIC en Colombia, evidenciando el crecimiento en el número de empresas y de teletrabajadores en Colombia.
Con los anteriores pasos se buscó cumplir los requisitos básicos de una revisión, los cuales plantean la necesidad de sintetizar la evidencia existente relativa a una temática y a su vez proveer un marco de antecedentes que permita posicionar nuevas actividades de investigación referentes a la temática (Kitchenham, 2004).

\section{RESULTADOS Y DISCUSIÓN}

En este apartado se presentan los resultados de la revisión de literatura, con base en los aspectos benéficos e impactos que el teletrabajo tiene en la calidad de vida de los trabajadores.

Tabla 1. Investigaciones sobre el impacto del teletrabajo

Table 1. Research on the impact of telework

\begin{tabular}{|c|c|c|c|}
\hline $\begin{array}{c}\text { Fuente y año de } \\
\text { publicación }\end{array}$ & $\begin{array}{l}\text { Objeto de la } \\
\text { investigación }\end{array}$ & Idea principal & Resultados \\
\hline $\begin{array}{l}\text { Teletrabajo y salud: } \\
\text { un nuevo reto para } \\
\text { la psicología } \\
\text { (Abregat y Gallego, } \\
\text { 2002) }\end{array}$ & $\begin{array}{l}\text { Identificar cuáles } \\
\text { pueden ser en el } \\
\text { futuro las } \\
\text { necesidades de } \\
\text { los } \\
\text { teletrabajadores, } \\
\text { de cara a } \\
\text { preservar su } \\
\text { salud, y } \\
\text { adaptación } \\
\text { funcionamiento } \\
\text { óptimo desde el } \\
\text { punto de vista de } \\
\text { la psicología. }\end{array}$ & $\begin{array}{l}\text { Revisión sobre aquellos } \\
\text { factores o } \\
\text { inconvenientes que } \\
\text { pueden incidir de } \\
\text { manera negativa o } \\
\text { positiva en la salud física } \\
\text { y psíquica de los } \\
\text { teletrabajadores, } \\
\text { teniendo en cuenta el } \\
\text { perfil ideal que deben } \\
\text { poseer para el desarrollo } \\
\text { eficiente de sus } \\
\text { actividades. El desajuste } \\
\text { en estas características } \\
\text { puede llevar, en última } \\
\text { instancia, a la afectación } \\
\text { de su salud. }\end{array}$ & $\begin{array}{l}\text { Las consecuencias psicológicas } \\
\text { pueden ser varias: emocionales, } \\
\text { cognitivas y conductuales, siendo la } \\
\text { palabra relevante «el estrés». Las } \\
\text { consecuencias del estrés pueden } \\
\text { implicar un conjunto complejo de } \\
\text { respuestas fisiológicas, que afectan } \\
\text { gravemente el bienestar y la calidad } \\
\text { de vida del que lo padece y le rodean. } \\
\text { Las consecuencias físicas pueden ser: } \\
\text { sedentarismo, obesidad por la } \\
\text { exposición permanente a los } \\
\text { alimentos, comportamientos } \\
\text { compulsivos, entre otras. }\end{array}$ \\
\hline $\begin{array}{l}\text { El teletrabajo: una } \\
\text { estrategia de } \\
\text { motivación (Vega y } \\
\text { Flórez, 2014) }\end{array}$ & $\begin{array}{l}\text { Demostrar que el } \\
\text { teletrabajo } \\
\text { podría } \\
\text { considerarse } \\
\text { como una de las } \\
\text { estrategias de } \\
\text { motivación, a } \\
\text { través de la cual } \\
\text { le permite a } \\
\text { quien lo ejerce, } \\
\text { obtener una } \\
\text { mayor calidad de }\end{array}$ & $\begin{array}{l}\text { La motivación es } \\
\text { esencial para el } \\
\text { desempeño individual } \\
\text { de un trabajador y el } \\
\text { comportamiento } \\
\text { organizacional de una } \\
\text { empresa, lo que trae } \\
\text { como consecuencia una } \\
\text { mayor satisfacción y } \\
\text { productividad para } \\
\text { ambas partes. Se citan } \\
\text { cinco teorías que se }\end{array}$ & $\begin{array}{l}\text { El teletrabajo motiva a que el } \\
\text { funcionario opte de forma voluntaria } \\
\text { a laborar desde su domicilio o } \\
\text { cualquier otro lugar distinto al de su } \\
\text { oficina. Para que se ejecute } \\
\text { correctamente, quien opte por esta } \\
\text { modalidad debe contar con siete } \\
\text { competencias fundamentales: } \\
\text { automotivación, autorregulación, } \\
\text { compromiso, comunicación, } \\
\text { aprendizaje, orientación a la calidad y } \\
\text { juicio. Trae como resultado una mejor }\end{array}$ \\
\hline
\end{tabular}




\begin{tabular}{|c|c|c|c|}
\hline & $\begin{array}{l}\text { vida, un mejor } \\
\text { manejo de su } \\
\text { tiempo, una } \\
\text { economía } \\
\text { considerable en } \\
\text { sus gastos, entre } \\
\text { otros. }\end{array}$ & $\begin{array}{l}\text { aproximan a lo que } \\
\text { podría pretender el } \\
\text { teletrabajo en una } \\
\text { organización y en los } \\
\text { individuos. }\end{array}$ & $\begin{array}{l}\text { calidad de vida en el teletrabajador y } \\
\text { un aumento en la productividad para } \\
\text { la empresa. La persona que haga } \\
\text { teletrabajo potencia sus habilidades y } \\
\text { capacidades, pues requiere ser } \\
\text { innovador y responsable. }\end{array}$ \\
\hline $\begin{array}{ll}\text { Los } & \text { riesgos } \\
\text { psicosociales en el } \\
\text { trabajo } \quad \text { (Rubini, } \\
2012 \text { ) }\end{array}$ & $\begin{array}{ll}\text { Identificar } & \text { los } \\
\text { principales } & \\
\text { factores } & \text { de } \\
\text { riesgo } & \\
\text { psicosocial } & \text { del } \\
\text { teletrabajo. } & \end{array}$ & $\begin{array}{l}\text { Los riesgos psicosociales } \\
\text { son aquellos factores } \\
\text { individuales, } \\
\text { organizacionales r y } \\
\text { relacionales } \\
\text { influyen que } \\
\text { desempeño de un } \\
\text { individuo. }\end{array}$ & $\begin{array}{l}\text { El teletrabajo puede exponer a los } \\
\text { trabajadores a serios e irreparables } \\
\text { riesgos sobre su salud psíquica y } \\
\text { relacional. Mediante un abordaje } \\
\text { teórico se identifican los principales } \\
\text { factores de riesgo psicosocial del } \\
\text { teletrabajo y las consecuencias para el } \\
\text { trabajador. }\end{array}$ \\
\hline $\begin{array}{l}\text { ¿Por qué estudiar } \\
\text { las relaciones } \\
\text { sociales en las } \\
\text { teleorganizaciones? } \\
\text { (Rubini, 2015) }\end{array}$ & $\begin{array}{l}\text { Revisar cómo se } \\
\text { establecerán las } \\
\text { relaciones } \\
\text { laborales en las } \\
\text { nuevas tele- } \\
\text { organizaciones. }\end{array}$ & $\begin{array}{l}\text { Con la globalización y el } \\
\text { desarrollo de las } \\
\text { tecnologías de la } \\
\text { información y la } \\
\text { comunicación aparecen } \\
\text { las tele-organizaciones y } \\
\text { el teletrabajo. Estos } \\
\text { implican cambios en los } \\
\text { modos de organización y } \\
\text { trabajo tradicionales. } \\
\text { Algunos, como los } \\
\text { cambios en la } \\
\text { estructura, la } \\
\text { coordinación la } \\
\text { actividades, el control } \\
\text { del trabajo y las } \\
\text { relaciones sociales en el } \\
\text { trabajo están siendo } \\
\text { estudiados por la } \\
\text { administración. }\end{array}$ & $\begin{array}{l}\text { Se argumenta por qué se considera de } \\
\text { suma importancia que la } \\
\text { Administración estudie cómo son las } \\
\text { relaciones sociales que se gestan y } \\
\text { sostienen en la organización cuando } \\
\text { sus integrantes teletrabajan. La } \\
\text { investigación se apoya en: 1) la } \\
\text { concepción del hombre como un ser } \\
\text { social; 2) la importancia que este } \\
\text { asigna a los vínculos sociales en el } \\
\text { trabajo; y 3) la concepción de la } \\
\text { organización como un sistema social } \\
\text { que para constituirse y mantenerse } \\
\text { en el tiempo requiere encontrar un } \\
\text { equilibrio entre sus intereses y los de } \\
\text { sus integrantes. }\end{array}$ \\
\hline $\begin{array}{l}\text { Teletrabajo y vida } \\
\text { cotidiana: Ventajas } \\
\text { y dificultades para la } \\
\text { conciliación de la } \\
\text { vida laboral, } \\
\text { personal y familiar } \\
\text { (Sánchez y Mozo } \\
\text { 2009) }\end{array}$ & $\begin{array}{l}\text { Analiza si el } \\
\text { teletrabajo se } \\
\text { constituye en } \\
\text { una estrategia de } \\
\text { conciliación de la } \\
\text { vida laboral, } \\
\text { familiar y } \\
\text { personal para las } \\
\text { mujeres } \\
\text { teletrabajadoras } \\
\text { con cargas } \\
\text { familiares. }\end{array}$ & $\begin{array}{l}\text { El discurso sobre el } \\
\text { teletrabajo no es } \\
\text { homogéneo, compacto } \\
\text { orineal. } \\
\text { Contradictoriamente: } \\
\text { libera y esclaviza, es una } \\
\text { trampa y una } \\
\text { oportunidad, realiza } \\
\text { personalmente y puede } \\
\text { significar una renuncia. } \\
\text { Pero, a pesar de esta rica } \\
\text { complejidad, los riesgos } \\
\text { y los posibles efectos } \\
\text { perniciosos del } \\
\text { teletrabajo no pueden } \\
\text { ensombrecer su } \\
\text { potencial laboral ni los } \\
\text { beneficios. }\end{array}$ & $\begin{array}{l}\text { A pesar de esta valoración positiva, el } \\
\text { miedo al impacto que el teletrabajo } \\
\text { pueda ejercer en el desarrollo de la } \\
\text { carrera dentro de la organización, } \\
\text { agravado por la condición de ser } \\
\text { mujer, es una de las desventajas } \\
\text { percibidas del teletrabajo. } \\
\text { El estudio demuestra que la } \\
\text { autogestión del tiempo es una de las } \\
\text { variables más importantes que } \\
\text { influyen en la decisión de teletrabajar; } \\
\text { el hecho de que la persona que } \\
\text { teletrabaja sea autónoma para } \\
\text { organizarse ella misma el tiempo de } \\
\text { trabajo le hace interiorizar un } \\
\text { sentimiento de "culpabilidad» (por } \\
\text { poder trabajar en mejores } \\
\text { condiciones, evitar tiempo de }\end{array}$ \\
\hline
\end{tabular}


transporte o escapar de las interrupciones de la oficina) que le lleva a trabajar más tiempo para compensarlo.

\begin{tabular}{ll}
\hline El enfoque de & Analizar \\
género aplicado a la & impacto \\
relación teletrabajo- & teletrabajo en \\
medio ambiente (De & medio ambiente \\
Luis, Martínez, & \\
Pérez y Vela, 2004) &
\end{tabular}

Pérez y Vela, 2004) el El principal efecto

directo del teletrabajo

sobre el medio

ambiente estaría en la

reducción del consumo

de energía y, por tanto,

en una menor emisión

de contaminantes a la

atmósfera como

consecuencia de la

reducción de los

desplazamientos

laborales de los

teletrabajadores a las

empresas.

\begin{tabular}{ll}
\hline El teletrabajo en & Entender el \\
América Latina y el & estado actual del \\
Caribe (Di Martino, & teletrabajo en \\
2004) & América Latina y \\
& el Caribe en \\
& países como \\
& Argentina, Brasil, \\
& Uruguay y \\
& México.
\end{tabular}

El teletrabajo tiene un impacto medioambiental positivo en el entorno urbano, al menos en el corto plazo. El impacto del teletrabajo en casa y en telecentros es el más estudiado y muestra una reducción significativa en el número de kilómetros recorridos y en la emisión de contaminantes.

El potencial del

teletrabajo en cuanto a seguir desarrollándose y

convertirse en un

fenómeno económico y social clave en ALC, depende de una serie de factores. Algunos de ellos, como la posición geográfica, constituyen factores invariables; otros, como la capacidad lingüística y la mano de obra educada, pueden variar solo en una perspectiva de mediano - largo plazo. Otros pueden estar más abiertos al cambio en un plazo relativamente corto y son los que pueden determinar el ritmo del desarrollo del teletrabajo. Entre estos últimos la difusión de las TIC desempeña un papel de gran importancia.
En los últimos años, el abordaje del teletrabajo en ALC se ha caracterizado en gran medida por un empuje hacia la innovación tecnológica, reducción de costos para aumentar la competitividad, búsqueda de una mayor flexibilidad laboral, reconfiguración de las organizaciones, lucha contra el desempleo, aprovechamiento de las oportunidades del mercado global. Este es el enfoque que se ha seguido acompañado de importantes desventajas: flexibilidad que se vuelve incontrolable, reducción que puede ser interpretada como algo malo, condiciones de trabajo que se deterioran y aumento de la precariedad del trabajo. Esto bajo la presunción de que las desventajas son de naturaleza temporaria, y que, eventualmente, los ciclos virtuosos se activarán y eso redundará en beneficios generales tanto para la industria como para las personas que trabajan.

Fuente: elaboración propia con base en los autores mencionados. 


\section{Beneficios del teletrabajo en la calidad de vida del trabajador}

El teletrabajo ha sido poco implementado en países en desarrollo, ya que las empresas y los empleados no conocen los diversos factores asociados a la puesta en marcha de esta modalidad laboral. Debido a lo anterior, se plantea la necesidad de impulsar el teletrabajo a partir de un examen sobre la percepción de la nueva fuerza laboral, con el fin de formular estrategias adecuadas que permitan un mayor acompañamiento desde el sector público y privado.

Entre los efectos destacables que tiene esta práctica está el manejo de situaciones laborales especiales, en las cuales el trabajador y/o su grupo familiar presentan problemas de salud o discapacidad o se necesite la atención por parte del trabajador a su grupo familiar por contar con adultos mayores o hijos pequeños. Así, el teletrabajo aumenta el control familiar y alivia la tensión laboral, manejando un equilibrio entre trabajo, funciones y roles familiares (Tremblay, 2003).

La implementación del teletrabajo tiene entre otros beneficios para las empresas como la sostenibilidad y adaptación a los cambios económicos y tecnológicos del mercado (Peters, Tijdens y Wetzels, 2004). Asimismo, ofrece ventajas competitivas al mejorar la flexibilidad que puede asociarse inicialmente a altos costos, situación que tiende a disminuir con el tiempo (Karia y Asaari, 2016). Esta flexibilidad puede ser funcional y numérica, por medio de subcontratación o por medio de empresas temporales que son intermediarias para realizar algunas funciones específicas (Martínez, Pérez, de Luis y Vela, 2007).

Por otro lado, permite la reducción de gastos para el empleado y de costos para la empresa, debido a que este puede desarrollarse en cualquier lugar y no es necesario un espacio físico (Cárcamo y Villegas, 2009). En este sentido, se ha encontrado que el teletrabajo aporta al desarrollo y protección del medio ambiente (Egbuta, Thomas y Al-Hasan, 2014), aumenta la innovación, estimula el uso de la tecnología web y de la estructura industrial de los países, la cercanía familiar (Kossek, Lautsch y Eaton, 2009), lo que favorece al crecimiento de sectores como la informática.

En materia de movilidad, en tanto que las personas laboran en sus casas disminuye ostensiblemente el flujo de vehículos (Pirdavani, Bellemans, Brijs, Kochan y Wets, 2015), mejorando la movilidad sin tener que acudir o ampliar las restricciones vehiculares que existen hoy en día como son el pico y placa en las principales ciudades de Colombia, y en caso de ser necesaria la restricción de movilidad vehicular, no se vería afectada la producción o cumplimiento de metas, puesto que empleados y trabajadores pueden desarrollar sus actividades y tareas desde su casa, el telecentro o el café internet apoyados en la tecnología.

Adicionalmente, el teletrabajo puede ser visto como un ejemplo de la flexibilización o destradicionalización del trabajo (Al-Jaafreh y Mosleh, 2011), donde lugares, contratos y horas de trabajo se abren para la renegociación, pues el teletrabajador es quien dispone de su tiempo cumpliendo con la productividad (Yttri, 1999; Van Lier, De Witte y Macharis, 2014). Es así como se obtiene un costo-beneficio para los empleados: el costo de un salario menor a un empleado fijo en la empresa, pero con el beneficio de ahorrar el tiempo y dinero que requieren los desplazamientos a un lugar fijo de trabajo (Vesala y Tuomivaara, 2015). Además, se argumenta que las mujeres se sienten más satisfechas con este ahorro que los hombres, a causa de las responsabilidades que tienen en su casa y con sus hijos (Hilbrecht, Shaw, Johnson y Andrey, 2008).

La aplicación del teletrabajo en una región determinada debe estudiarse desde la historia, la cultura y la educación, puesto que a partir de estos aspectos se puede concluir acerca de la 
eficiencia de esta modalidad laboral (Mitter, 2000). Como nueva tendencia laboral, el teletrabajo es adoptado e influenciado por factores económicos, políticos, sociales y tecnológicos, entre otros. No obstante, el transporte, la calificación de la mano de obra, los sindicatos pueden ser una barrera organizativa para su desarrollo e implementación. Los países con alta densidad de población tienen mayor cantidad de teletrabajadores, aunque en algunas ocasiones puede pasar lo contrario por factores específicos del país o región (Tolbert y Zucker, 1983, DiMaggio, 1988, citados en Daniels, Lamond y Standen, 2001).

En el ámbito de la gestión del talento humano, las empresas que implementan el teletrabajo manejan horarios flexibles, sus empleados se evalúan por resultados y en ocasiones se utiliza la compensación variable. Sin embargo, es importante considerar que es diferente la flexibilidad dada por el teletrabajo a la obtenida por la subcontratación eventual (Haddon y Lewis 1994; Martínez et. al., 2007). Por esta razón, el teletrabajo es aplicable principalmente en empresas que prestan servicios o donde sus tareas se pueden realizar por medio de datos con el uso de las TIC (Daniels, Lamond y Standen, 2001; Ramírez y Rúa, 2014).

\section{Posibles impactos negativos del teletrabajo en la calidad de vida laboral.}

Algunas empresas interesadas solo en la productividad y no en las relaciones sociales o satisfacción del trabajador utilizan el teletrabajo con el objetivo de minimizar el tiempo laborado (Hörning, Gerhard y Michailow, 1995). Pese a los resultados positivos, se han identificado desventajas del teletrabajo entre las que se destaca la disminución de la calidad de vida laboral, la baja participación e interacción con el personal de la organización, el poco sentido de pertenencia y en ocasiones menor posibilidad de ascenso y proyección laboral. Asimismo, el teletrabajo aparta la fuerza afectiva y lazos de afectos en el contexto laboral, pues no hay tanta oportunidad de acercarse y compartir con los compañeros de trabajo (Cárcamo y Villegas, 2009; Abdullah y Ismail, 2012; Scott, Dam, Páez y Wilton, 2012).

Al respecto, tal como plantea la regulación en esta materia, las empresas deben garantizar desde la gestión del talento humano que se formulen estrategias para evitar ocasionar estrés, baja productividad, dificultad de definir con claridad los resultados esperados de un trabajo, falta de alineamiento de estilos de trabajo en los equipos y falta de integración social y participación en las actividades de la empresa

En materia de relaciones laborales y la sindicalización no favorece el desarrollo del teletrabajo, puesto que la función principal del sindicato o las asociaciones sindicales es aportar una voz colectiva de los empleados que vienen a trabajar en un sitio determinado, no de los empleados que laboran fuera de la empresa. Se objeta que la modalidad de contrato y el salario de un teletrabajador es diferente, no obstante, puede ser modificado en cualquier momento por el empleador para aceptar el regreso del teletrabajador a la planta física (Mitter, 2000).

Al respecto, se ha señalado que la modalidad de teletrabajo implica menores condiciones laborales y salarios más bajos que los ofrecidos a los trabajadores de planta en la empresa. Frente a esto, en Colombia existe la figura de la voluntariedad para realizar esta práctica, por lo cual el empleador debe exponer las ventajas, desventajas y características para que el empleado acepte o rechace esta modalidad laboral; esta característica ha hecho que los teletrabajadores se sientan satisfechos con su empleo (Betancourt, 2013; Ramírez et al., 2014). 


\section{Alineación estratégica: teletrabajo y talento humano}

La gestión estratégica del talento humano se ha desarrollado significativamente en las últimas dos décadas. En un principio, para la comprensión de la relación entre la gestión humana y el rendimiento de la empresa (Delery y Doty, 1996; Kramar, 2014) y posteriormente, debido a su fase evolutiva para identificar las necesidades estratégicas de las organizaciones, así como el desarrollo del talento necesarios para implementar una estrategia competitiva y el logro de los objetivos operativos y funcionales (Huselid, Jackson y Schuler, 1997).

La gestión del talento humano en las organizaciones es considerada como la combinación de procesos y resultados en las que las habilidades y la motivación de los empleados facilitan la vinculación de las funciones con los objetivos estratégicos, por lo que se ha constituido en un esfuerzo por mejorar el rendimiento de la empresa mediante la comprensión de la cultura organizacional, el compromiso y la participación de los empleados (Bamberger y Meshoulam, 2000).

En sus inicios, la gestión del talento humano surgió como una disciplina enfocada en la contribución estratégica de los empleados para el éxito de la organización sobre la necesidad de considerar el diseño de estrategias que permitiera integrar las habilidades de trabajo, intercambio de conocimientos, cooperación y el aporte de los empleados; sobre la base de un conjunto de prácticas consistentes con las tendencias y formas de trabajo que asegurara la contribución del capital humano a la consecución de los objetivos de organizacionales (Gratton, Hope-Hailey, Stiles, y Truss, 1999; Jackson y Schuler, 1995; Ugheoke, Isa y Noor, 2014).

Actualmente es posible evidenciar una serie de enfoques de la gestión humana que pueden ser utilizados por las organizaciones para responder a los desafíos contemporáneos y desarrollar una cultura de competitividad. Al respecto, Wrigth, Dunford y Snell (2001) proponen el modelo conceptual del recurso humano en relación a su entorno desde las siguientes características: 1) el grupo humano que dispone la empresa, sus conocimientos y habilidades profesionales representan un stock de recursos que ha de ajustarse en cada momento a las necesidades estratégicas de la empresa; 2) el comportamiento cognitivo y relacional de los individuos y de los equipos, comprometido en el éxito de la empresa (MacDuffie, 1995); y 3) el sistema de gestión estratégica de personal, que integra diferentes prácticas: diseño de tareas y responsabilidades, entrenamiento, sistemas de retribución basados en el desempeño.

En este mismo orden se evidencian otras prácticas alrededor de la gestión humana: 1) aquellas que buscan la inserción de los recursos humanos: reclutamiento, análisis y configuración de puestos y gestión por competencias; 2 ) las de desarrollo y promoción de los recursos humanos en la empresa: formación, mejora de contenidos y aprendizaje de la organización; 3) las que promueven la valoración del rendimiento y sistemas de compensaciones que permitan una retribución contingente a resultados: dirección por objetivos, incentivos y participación en resultados; 4) las de integración de la planificación estratégica de los recursos humanos en la planificación de los negocios: estrategias y objetivos específicos, es decir, el control integrado de la gestión de recursos humanos; y 5) los mecanismos sociales para integrar el comportamiento de los recursos humanos con los fines de la organización: comunicación interna o clima laboral (Sheppeck y Militello, 2000).

No obstante, en las nuevas tendencias de trabajo, como el trabajo en red o teletrabajo, no se identifican marcos conceptuales establecidos sobre los procesos claves de la teoría y la práctica de gestión humana (Taylor et al., 2015; Hollenbeck y Jamieson, 2015; 
Mitchell, Obeidat y Bray, 2013). Así, la incorporación de nuevas tecnologías trae consigo transformaciones en los estilos de vida de las personas, las prácticas de consumo, las relaciones interpersonales y por consiguiente cambios en las condiciones de supervivencia y competitividad de las empresas.

Lo anterior implica proveer nuevas estructuras para que la organización pueda afrontar y anticiparse a los cambios, como el elemento diferenciador del siglo XXI (Dolan, Cabrera, Jackson y Schuler, 2003), donde se destaca la eficacia de la estructura humana; es decir, la satisfacción de objetivos personales y de la organización (Baber y Waymon, 2010). En este sentido, algunas investigaciones indican que para los empleados más jóvenes es importante la vida social y optan por el trabajo tradicional, esto se evidencia en los procesos de selección para vacantes en teletrabajo (Haddon y Lewis, 1994; García, Martín y Sánchez, 2014).

Por otro lado, cabe destacar que la intervención política para el uso del teletrabajo no es muy notoria; esto se debe al alcance y perspectiva que toman las empresas para implementar este modelo de trabajo, pues influyen factores culturales y tecnológicos que hacen que la propagación de la práctica no sea efectiva para la organización (Mitter, 2000; Peters et al., 2004). Por último, es importante mencionar que una alternativa para incentivar el teletrabajo y manejo de las TIC puede ser mediante el pago de impuestos donde las empresas pueden tener reducción de impuestos por aplicar este modelo (European Commission, 1998, citado en Daniels, Lamond y Standen, 2001).

\section{CONCLUSIONES}

En Colombia, las empresas están mirando con mucho interés esta modalidad de trabajo por todos los beneficios que pueden significar para las personas, la empresa y la sociedad en las que se resalta la generación de empleo, el aumento de la formalización laboral, la reducción de costos operacionales, el aprovechamiento del tiempo, potencial de desarrollo e inclusión para mujeres, jóvenes, discapacitados y la mejora en la calidad de vida de la clase trabajadora, por mencionar algunos. Estos beneficios sin lugar a dudas son ideales compatibles con el objeto de coordinación económica y equilibrio social que reviste las normas laborales $y$, por lo tanto, debe en conjunto promover el desarrollo del teletrabajo; es indudable el atractivo de este entorno para los trabajadores y las empresas.

Tanto la flexibilidad horaria como una adecuada organización de la jornada laboral se convierten en las variables que, bien gestionadas, sirven para lograr un mayor compromiso y responsabilidad del trabajador con los objetivos empresariales $y$, por tanto, una mayor productividad, un alto grado de conciliación laboral y familiar y, por supuesto, un menor absentismo en la empresa. Por el contrario, una mala organización empresarial del tiempo de trabajo tiene como consecuencia un efecto negativo, no solo para el trabajador debido a la insatisfacción laboral y los cuadros de estrés, sino también para la empresa, pues la rigidez, tanto horaria como de las jornadas de trabajo, tienen una directa vinculación con el aumento de bajas por incapacidad temporal con sus consecuencias directas (Betancourt, 2013). Es por esto que, desde la perspectiva del individuo, respecto a la salud ocupacional, debe conllevar a promover políticas y disposiciones normativas, con el objetivo de generar mejores condiciones laborales.

En el contexto general es posible interpretarse que las dificultades para implementar el teletrabajo pueden estar cimentadas en la ausencia de la normatividad, pues es de reconocimiento mundial el avance tecnológico, las redes de comunicación y la Internet. El teletrabajo se asocia a las políticas de I+D+i por el aprovechamiento del avance tecnológico del país derivado de la apropiación de las TIC por parte de las entidades del sector público y privado. 
En consecuencia, el teletrabajo aporta a los procesos de innovación organizacional, aumento de la productividad, calidad de vida de los trabajadores, protección del medio ambiente, mejoramiento de la movilidad en las ciudades y la generación de procesos de calidad frente a los compromisos con el mercado internacional de bienes y servicios. No obstante, cada vez más está siendo cuestionado el rol doméstico y familiar del teletrabajador, que a pesar de la autonomía para manejar «su tiempo», finalmente no cuenta con él para asumir realmente su papel en el contexto individual, organizacional y social.

\section{REFERENCIAS}

Abdullah, H. y Ismail, N. (2012). Quality of work and life balance in teleworking. International Business Management, 6(2), 119-130. doi:10.3923/ibm.2012.119.130

Abregat, M. B. y Gallego, E. C. (2002). Teletrabajo y salud: un nuevo reto para la Psicología. Papeles del psicólogo, 83, 5561.

Al-Jaafreh, A. y Mosleh, F. (2011). The appropriateness of the organizational factors for the adoption of teleworking application the context of Mobile Telecommunications Sector. European Journal of Scientific Research, 57(4).

Baber, A. y Waymon, L. (2010). The connected employee: The 8 networking competencies for organizational success. $T+D, 64(2), 50$ 53.

Bamberger, P. y Meshoulam, I. (2000). Human resource management strategy. London: Published Sage.

Betancourt, R. B. (2013). Actualidad del teletrabajo en Colombia. Escuela Internacional de Alta Formación en
Relaciones Laborales y de Trabajo de ADAPT, 1(3).

Blanco, M. B. y Gil, R. J. (2014). Proposal for a call center to provide professional services via teleworking. Paper presented at the 4ta. Conferencia Iberoamericana En Sistemas, Cibernética e Informática, $\mathrm{CISCl}$ 2005, Memorias, 1, 278-282.

Brosveet, J. (1997). Public Sector Case Study of a Norwegian IT Highway Project. Trondheim, Centre for Technology and Society (SLIM working Paper No. 3).

Cárcamo, L. y Villegas, Ó. M. (2009). Competencias comunicativas y actitud hacia el teletrabajo de estudiantes universitarios chilenos. Revista Latina de Comunicación Social, 12(64).

Colombia, C. D. (2008). Ley 1221 de 2008. Normas para promover y regular el Teletrabajo. Bogotá, D.C.

Colombia, C. D. (2012). Decreto 884 de 2012 (s.f.). Regula aspectos de la Ley 1221 de 2008 para el Teletrabajo. Bogotá. Recuperado de http://www.alcaldiabogota.gov.co/sisjur/n ormas/Norma1.jsp?i=47216

Colombia, E. A. (2013). Ministerio de las Tecnologías de la Información y de las Comunicaciones.http://teletrabajo.mintic. gov.co/libroblanco/wpcontent/uploads/20 13/11/libro_completo_blanco-alta.pdf

Ministerio del Trabajo (2013). Guía jurídica de implementación del teletrabajo. Bogotá, D.C. mayo de 2013. ISBN: 978-958-871741-8

Ministerio de Tecnologías de la Información y las Comunicaciones -MinTIC- (2016). Recuperado de http://www.elespectador.com/noticias/ec onomia/cuatro-anos-se-triplicaron-losteletrabajadores-colombi-articulo-652107 
Corporación Colombia Digital (2012). Recuperado de http://colombiadigital.net/actualidad/noti cias/item/4122-

gu\%C3\%ADasjur\%C3\%ADdicas-y-deseguridad-laboral-para-implementar-elteletrabajo.html

Daniels, K.; Lamond, D. y Standen, P. (2001). Teleworking: frameworks for organizational research. Journal of Management Studies, 38(8), 1151-1185.

Delery, J. E. y Doty, D. H. (1996). Modes of theorizing in strategic human resource management: Tests of universalistic, contingency, and configurational performance predictions. Academy of management Journal, 39(4), 802-835.

Dolan, C., Jackson y Schuler (2003). La gestión de los recursos humanos: preparando profesionales para el siglo XXI. Ed. McGrawHill, Madrid.

Egbuta, I. C.; Thomas, B. y Al-Hasan, S. (2014). The Contribution of Teleworking towards a Green Computing Environment. Green Technology Applications for Enterprise and Academic Innovation, 218.

García, N.; Martín, F. y Sánchez, G. (2014). The moderating role of employees' perception on strategic human resource management and its influence on organizational performance. Revista Europea de Dirección y Economía de la Empresa, 23(3), 137-146. doi: 10.1016/j.redee.2014.03.002

Gibb, S. (2001). The state of human resource management: evidence from employees' views of HRM systems and staff. Employee Relations, 23(4-5), 318-336.

Gratton, L.; Hope-Hailey, V.; Stiles, P. y Truss, C. (1999). Linking individual performance to business strategy: The people process model. Human Resource
Haddon, L. y Lewis, A. (1994). The experience of teleworking: an annotated review. International Journal of Human Resource Management, 5(1), 193-223.

Hilbrecht, M.; Shaw, S. M.; Johnson, L. C. y Andrey, J. (2008). 'I'm home for the kids': contradictory implications for work-life balance of teleworking mothers. Gender, Work \& Organization, 15(5), 454-476.

Hollenbeck, J. y Jamieson, B. (2015). Human capital, social capital, and social network analysis: Implications for strategic human resource management. Academy of Management Perspectives, 29(3), 370-385. doi:10.5465/amp.2014.0140

Hörning, K. H.; Gerhard, A. y Michailow, M. (1995). Time pioneers: Flexible working time and new lifestyles. Cambridge: Polity Press.

Huselid, M. A.; Jackson, S. E. y Schuler, R. S. (1997). Technical and strategic human resources management effectiveness as determinants of firm performance. Academy of Management journal, 40(1), 171-188.

Jackson, S. E. y Schuler, R. S. (1995). Understanding human resource management in the context of organizations and their environments. Human Resource Management: Critical Perspectives on Business and Management, 2, 45-74.

Julsrud, T. E. (2010). Emergent use of video conferences among business travellers: Norwegian experiences. Videoconferencing, changing behaviour of business travellers and its effects on the aviation market

Karia, N. y Asaari, M. H. (2016). Innovation capability: the impact of teleworking on sustainable competitive 
advantage. International Journal of Technology, Policy and Management, 16(2), 181-194.

Kitchenham, B. (2004). Procedures for performing systematic reviews. Keele University, 33.

Kossek, E. E.; Lautsch, B. A. and Eaton, S. C. (2009). Good Teleworking: Under what conditions does teleworking enhance employees' well-being. Technology and Psychological Well-Being. Cambridge University Press,148-173.

Kramar, R. (2014). Beyond strategic human resource management: Is sustainable human resource management the next approach? International Journal of Human Resource Management, 25(8), 1069-1089. doi:10.1080/09585192.2013.816863

Kuvaas, B. y Dysvik, A. (2010). Exploring alternative relationships between perceived investment in employee development, perceived supervisor support and employee outcomes. Human Resource Management Journal, 20(2), 138-156.

Lévy-Leboyer, C. (2003). Gestión de las competencias. Cómo analizarlas, cómo evaluarlas, cómo desarrollarlas. Barcelona: Ediciones Gestión 2000.

MacDuffie, J. P. (1995). Human resource bundless and manufacturing performance: organizational logic and flexible production systems in the world auto industry. Industrial and Labor Relations Rev. 48(2), 197-221.

Meroño-Cerdán, A. L. (2016). Perceived benefits of and barriers to the adoption of teleworking: peculiarities of Spanish family firms. Behaviour \& Information Technology, 1-12.
Mitchell, R.; Obeidat, S. y Bray, M. (2013). The effect of strategic human resource management on organizational performance: The mediating role of highperformance human resource practices. Human Resource Management, 52(6), 899-921. doi:10.1002/hrm.21587

Mitter, S. (2000). Teleworking and teletrade in India: combining diverse perspectives and visions. Economic and Political Weekly, 2241-2252.

Moreno, A.; Uriarte, L. M.; Mahou, Á. \& Álvarez, M. (2013). Mejora de la productividad de trabajadores del conocimiento. Big Data, 95, 126.

Muñoz-Doyague, M. F. y Nieto, M. (2012). Individual creativity performance and the quality of interpersonal relationships. Industrial Management \& Data Systems, 112(1), 125-145.

Peters, P.; Tijdens, K. and Wetzels, C. (2004), Employees' opportunities, preferences, and practices in telecommuting adoption. Information and Management, 41(4), 46982.

Pirdavani, A.; Bellemans, T.; Brijs, T.; Kochan, B. y Wets, G. (2015). Traffic safety implications of travel demand management policies: The cases of teleworking and fuel cost increase. Transportation systems and engineering: Concepts, methodologies, tools, and applications. doi:10.4018/978-14666-8473-7.ch055

Ramírez, S. y Rúa, N. (2014). El concepto de teletrabajo: aspectos para la seguridad y salud en el empleo. Revista CES Salud Pública, 5(1), 82-91.

Rodríguez, R.; Guest, D.; Oliveira, T. y Alfes, K. (2015). Who benefits from independent careers? employees, organizations, or 
both? Journal of Vocational Behavior, 91, 23-34. doi: 10.1016/j.jvb.2015.09.005

Martínez, Pérez, de Luis \& Vela (2007). Teleworking and workplace flexibility: a study of impact on firm performance. Personnel Review, 36(1), 4264.

Sánchez, C. P. y Mozo, A. M. (2009). Teletrabajo y vida cotidiana: ventajas y dificultades para la conciliación de la vida laboral, personal y familiar. Athenea Digital. Revista de pensamiento e investigación social, 15, 57-79.

Scott, D.; Dam, I.; Páez, A. y Wilton, R. (2012). Investigating the effects of social influence on the choice to telework. Environment and Planning A, 44(5), 1016-1031. doi:10.1068/a43223

Sheppeck, M. y Militello, J. (2000). Strategic HR configurations and organizational performance. Human Resource Management, 39(1), 5-16.

Stavrou, E. y Brewster, C. (2005). The configurational approach to linking strategic human resource management bundles with business performance: Myth or reality. Management Review, 16(2), 187-201.

Tahavori, Z. (2014). The Survey of Viewpoint of Teleworkers in Department of Information Processing about Advantages and Disadvantages of Teleworking. Journal of Information processing and Management, 28(4), 1069-1101.

Taylor, T.; Doherty, A. y McGraw, P. (2015). Managing people in sport organizations: A strategic human resource management perspective (Second edition). doi:10.4324/9781315881881
Tregaskis, O. (2000). Telework in its national context. Managing telework, 9-20.

Tremblay, D. G. (2003). Telework: a new mode of gendered segmentation? Results from a study in Canada. Canadian Journal of Communication, 28(4).

Ugheoke, S. O.; Isa, M. F. y Noor, W. S. (2014). Assessing the Impact of Strategic Human Resource Management on Tangible Performance: evidence from Nigerian SMEs. International Review of Management and Business Research, 3(2).

Van Lier, T.; de Witte, A. y Macharis, C. (2014). How worthwhile is teleworking from a sustainable mobility perspective? the case of brussels capital region. European Journal of Transport and Infrastructure Research, 14(3), 244-267. Retrieved from www.scopus.com

Vega, A. O. y Flórez, N. V. (2014). El teletrabajo: una estrategia de motivación. Revista Nacional de Administración, 5(2), 41-56.

Verburg, R.; Den Hartog, D. y Koopman, P. (2007). Configurations of human resource management practices: A model and test of internal fit. The International Journal of Human Resource Management, 18(2), 184-208.

Vesala, H. y Tuomivaara, S. (2015). Slowing work down by teleworking periodically in rural settings? Personnel Review, 44(4), 511-528.

Wright, P.; Dunford, B. y Snell, S. (2001). Human Resources and the resource-based view of the firm. Journal of Management, 27. 701-727.

Yttri, B. (1999). Homework and boundary work. Feature Special, 39. 